\section{Cataract surgery: benchmarks for established and trainee surgeons}

JM Sparrow ${ }^{1,2}$

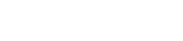

Eye (2008) 22, 1371-1372; doi:10.1038/eye.2008.282; published online 12 September 2008

Much attention is currently focused on surgical outcomes, with policy clearly in favour of the publication of individual results for consultant surgeons. ${ }^{1-3}$ Approximately a quarter of cataract surgery in the NHS is, however, undertaken by trainees at specialist registrar (SpR) level, ${ }^{4}$ which amounts to around 75000 operations annually in England alone. It is accepted that posterior capsule rupture (PCR) rates for learners are higher than those for experienced phacoemulsification surgeons - an unsurprising finding. ${ }^{5,6}$ With this number of procedures being undertaken in the NHS by trainees, it is a matter of public health relevance to know what complication rates should be expected for this group of training surgeons.

A recent electronic audit of 55567 cataract operations identified an overall PCR rate of $1.92 \%$ for 406 surgeons of all grades working in the English NHS. ${ }^{4}$ Analysis of risk factors for PCR revealed surgeon's grade to be an important determinant of complication risk. ${ }^{7}$ Trainees at SpR level were $65 \%$ more likely to have a complication than consultants, after adjustment for other risk factors. A major determinant of PCR risk was case complexity, patients with multiple risk factors being at a strikingly increased risk of a complication as predicted by the logistic regression model. A male patient aged above 90 years with diabetic retinopathy and a brunescent cataract, for example, would have a predicted probability of a PCR of $10 \%$ if operated on by a consultant, but around $16 \%$ if operated on by an SpR. This difference in predicted probability of a complication of $6 \%$ between the surgeons makes a strong case for such higher risk patients being operated on exclusively by consultants.

In this issue Buchan and Cassels-Brown ${ }^{8}$ have provided information on surgical opportunities, and surgical complication rates for trainees at
SpR level in the Yorkshire region. Surgical opportunities appeared adequate in this sample of trainees, and assuming representativeness, the finding bodes well for the training opportunities of the next generation of consultant ophthalmologists. The overall PCR rate for the 19 participating trainees of $2.3 \%$ is also reassuring. Without information on surgical case mix, however, the full picture remains uncertain. If the case mix complexity of these trainee surgeons was such that they operated exclusively on the lowest risk patients, then from the logistic regression model noted above, ${ }^{7}$ the baseline predicted probability of such a case mix would be around $0.75 \%$ with a consultant operating and around $1.2 \%$ with a SpR operating. Clearly, no audit series of over 4000 operations could exist where all cases were at baseline risk in terms of case complexity, but the figures serve to illustrate how crucially dependent these complication rates are on surgical case mix.

Personal audit of outcomes for individual surgeons becomes meaningful only when case complexity is taken into account. An excellent surgeon may take on challenging surgical cases and have raw outcome figures which are average or worse than average. Once case mix is taken into account his or her outcome figures may appear entirely different, reflecting more accurately the true surgical skill of the individual. There are concerns that publication of unadjusted figures may induce risk aversive behaviour, thus denying needy patients with potentially much to gain the opportunity of undergoing and benefiting from surgery. Without appropriate risk adjustment, unintended distortions of well-judged surgical practice may thus arise to the ultimate detriment of the public. In terms of surgical learning, a combination of case mix adjustment and adjustment for stage of training would be needed to correctly understand training progress. These various factors can be taken into
${ }^{1}$ Department of Ophthalmology, Bristol Eye Hospital, Lower Maudlin Street, Bristol BS1 2LX, UK

${ }^{2}$ International Centre for Eye Health, London School of Hygiene and Tropical Medicine, Keppel Street, London WC1E 7HT, UK

Correspondence: JM Sparrow,

Ophthalmology, Bristol Eye Hospital, Lower Maudlin Street, Bristol

BS1 2LX, UK

Tel: + 0117928 4697; Fax: 01179284686 E-mail: John.Sparrow@ doctors.org.uk 
account by application of an appropriate methodology as illustrated by the examples above. Calculations around risk are necessary and not overly complex once the model is established. In the era of electronic patient record keeping such calculations should not present a barrier. Competent surgeons of all grades should have nothing to fear from publication of properly audited and risk-adjusted outcomes.

\section{References}

1 Keogh B, Kinsman R. Fifth National Adult Cardiac Surgical Database Report (5th Blue Book). Improving outcomes for patients. 2003. http://www.scts.org/sections/audit/ Cardiac/index.html.

2 Keogh B, Spiegelhalter D, Bailey A, Roxburgh J, Magee P, Hilton C. The legacy of Bristol: public disclosure of individual surgeons' results. BMJ 2004; 329: 450-454.

3 Keogh B. Outcome measurement in surgery: The case, the tools and the timing. Oral presentation at the Royal College of Surgeons, 27 May 2008.
4 Jaycock P, Johnston RL, Taylor H, Adams M, Tole DM, Galloway $\mathrm{P}$ et al. The Cataract National Dataset electronic multi-centre audit of 55567 operations: updating benchmark standards of care in the United Kingdom and internationally. Eye; e-pub ahead of print 2007.

5 Bhagat N, Nissirios N, Potdevin L, Chung J, Lama P, Zarbin MA et al. Complications in resident-performed phacoemulsification cataract surgery at New Jersey Medical School. Br J Ophthalmol 2007; 91: 1315-1317.

6 Quillen DA, Phipps SJ. Visual outcomes and incidence of vitreous loss for residents performing phacoemulsification without prior planned extracapsular cataract extraction experience. Am J Ophthalmol 2003; 135: 732-733.

7 Narendran N, Jaycock P, Johnston RL, Taylor H, Adams M, Tole DM et al. The Cataract National Dataset electronic multicentre audit of 55567 operations: risk stratification for posterior capsule rupture and vitreous loss. Eye; e-pub ahead of print 2008.

8 Buchan J, Cassels-Brown A. Determinants of cataract surgical opportunities and complication rates in UK higher specialist training. Eye; current issue 2008. 